\title{
Distinguishing multiple-merger from Kingman coalescence using two-site frequency spectra
}

\author{
Daniel P. Rice ${ }^{1,2}$, John Novembre ${ }^{1,2, *}$, and Michael M. Desai ${ }^{3,4, *}$ \\ ${ }^{1}$ Department of Human Genetics, University of Chicago, Chicago, IL \\ ${ }^{2}$ Department of Ecology and Evolution, University of Chicago, \\ Chicago, IL \\ ${ }^{3}$ Department of Organismic and Evolutionary Biology, Harvard \\ University, Cambridge, MA \\ ${ }^{4}$ Department of Physics, Harvard University, Cambridge, MA \\ ${ }^{*}$ These authors contributed equally to this work.
}

November 3, 2018

\begin{abstract}
Demographic inference methods in population genetics typically assume that the ancestry of a sample can be modeled by the Kingman coalescent. A defining feature of this stochastic process is that it generates genealogies that are binary trees: no more than two ancestral lineages may coalesce at the same time. However, this assumption breaks down under several scenarios. For example, pervasive natural selection and extreme variation in offspring number can both generate genealogies with "multiple-merger" events in which more than two lineages coalesce instantaneously. Therefore, detecting multiple mergers is important both for understanding which forces have shaped the diversity of a population and for avoiding fitting misspecified models to data. Current methods to detect multiple mergers in genomic data rely on the site frequency spectrum (SFS). However, the signatures of multiple mergers in the SFS are also consistent with a Kingman coalescent with a time-varying population size. Here, we present a new method for detecting multiple mergers based on the pointwise mutual information of the two-site frequency spectrum for pairs of linked sites. Unlike the SFS, the pointwise mutual information depends mostly on the topologies of genealogies rather than on their branch lengths and is therefore largely insensitive to population size change. This statistic is global in the sense that it can detect when the genome-wide
\end{abstract}


genetic diversity is inconsistent with the Kingman coalescent, rather than detecting outlier regions, as in selection scan methods. Finally, we demonstrate a graphical model-checking procedure based on the pointwise mutual information using genomic diversity data from Drosophila melanogaster.

\section{Introduction}

The genetic diversity of a population reflects its demographic and evolutionary history. Learning about this history from contemporary genetic data is the domain of modern population genetics (see Hahn 2018). The fundamental tools of the trade are simplified mathematical models, which connect unobserved quantities such as the population size to observable features of genetic data. However, populations are complicated and, moreover, vary in their complications. No simple model can capture the processes governing every species' evolution, and a misspecified model will generate misleading inferences. It is therefore crucial to understand the limits of population genetics models and to assess when a model is appropriate for a particular data set.

One of the most widely used models is the Kingman coalescent (Kingman 1982b; Kingman 1982a; Hudson 1983; Tajima 1983). The Kingman coalescent is a stochastic process that generates gene genealogies: trees representing the patterns of shared ancestry of sampled individuals. Inference methods use these genealogies as latent variables linking demographic parameters to genetic data (Rosenberg and Nordborg 2002). The Kingman coalescent has a number of convenient properties that facilitate both analytical calculations (e.g., Tajima 1989) and efficient stochastic simulations (e.g., Hudson 2002): tree topologies are independent of waiting times; waiting times are generated by a Markov process; and neutral mutations are modeled as a Poisson process conditionally independent of the tree. Moreover, the model can be extended to study a variety of biological phenomena including recombination, population structure, and variation in sex ratios or ploidy (see generally Wakeley 2009).

An important application of the Kingman coalescent is inferring historical population sizes from genetic data (Schraiber and Akey 2015). In its simplest form, the model has a single parameter, the coalescent rate, which determines the branch lengths of genealogies (Kingman $1982 b)$. Under many conditions, the coalescent rate is inversely proportional to the population size (Kingman 1982a). Accordingly, a growing or shrinking population may be modeled by a time-varying rate (Griffiths and Tavaré 1994; Griffiths and Tavaré 1998). Patterns of genetic diversity depend on the ratio of the coalescent rate to other evolu- 
tionary rate parameters. For example, the site frequency spectrum (SFS) - the number of mutations segregating at different frequencies in a sample - is determined by the ratio of the mutation rate to the (time-varying) coalescent rate. Kingman coalescent-based inference methods solve the inverse problem of determining the population size history that best explains particular features of the data, such as the SFS (e.g., Bhaskar et al. 2015) or variations in heterozygosity along a chromosome (e.g., Li and Durbin 2011).

A serious problem for this class of inference methods is that different models of evolution generate different relationships between historical population sizes and genetic diversity. For example, one of the basic assumptions of the Kingman coalescent is that natural selection is negligible in determining the distribution of genealogies. When this assumption is violated, Kingman-based inference methods are misspecified. For instance, when a beneficial mutation increases rapidly in frequency, it distorts the genealogies at nearby sites (see e.g., Coop and Ralph 2012). If these "selective sweeps" occur regularly, they may be the dominant factor determining the distribution of genealogies. In this case, the average coalescent rate is proportional to the number of beneficial mutations introduced per generation, which is itself $d i$ rectly, rather than inversely, proportional to the population size. It follows that the relationship between the population size and the expected number of neutral mutations in a sample is inverted: larger populations will be less diverse than smaller populations.

While the example above is extreme, it is well established that violations of the neutrality assumption can distort or mask the signatures of population size changes. For example, Schrider et al. (2016) recently demonstrated that several popular inference methods give misleading results in the presence of selective sweeps. In a similar vein, Cvijović et al. (2018), showed that reduction of genetic diversity by purifying selection is accompanied by distortions in the SFS, leading to a false signal of population growth. Moreover, genomic evidence from multiple species suggests that such violations of neutrality may be widespread (Sella et al. 2009; Corbett-Detig et al. 2015; Kern and Hahn 2018).

An important extension of the Kingman coalescent is a family of models known as multiple-merger coalescents (Pitman 1999; Sagitov 1999; Donnelly and Kurtz 1999; reviewed in Eldon 2016), which arise in a variety of contexts both with and without selection. Whereas in the Kingman coalescent lineages may coalesce only pairwise, multiplemerger coalescents permit more than two lineages to coalesce in a single event. The more general class of simultaneous-multiple-merger coalescents (Schweinsberg 2000; Möhle and Sagitov 2001; Sagitov 2003) permits more than one distinct multiple-merger event at the same time. multiple-merger and simultaneous-multiple-merger models are relevant for species with "sweepstakes" reproductive events (Eldon and Wakeley 
2006; Sargsyan and Wakeley 2008), fat-tailed offspring number distributions (Schweinsberg 2003), recurring selective sweeps at linked sites (Durrett and Schweinsberg 2005; Coop and Ralph 2012), rapid adaptation (Neher and Hallatschek 2013; Desai et al. 2013), and purifying selection at sufficiently many sites (Seger et al. 2010; Nicolaisen and Desai 2012; Good et al. 2014).

In each of these contexts, the coalescent timescale is not necessarily proportional to the population size. For example, with fat-tailed offspring distributions the rate of coalescence is a power law in the population size (Schweinsberg 2003), while with linked sweeps it is determined by the rate of linked sweeps, as described above (Durrett and Schweinsberg 2005). In these settings, interpreting the level of genetic diversity in terms of an "effective population size" is misleading, and inferences based on the Kingman coalescent may be qualitatively incorrect.

It is therefore important to determine whether the Kingman model is appropriate for a given data set before performing demographic inference. This task is distinct from "selection scan" methods designed to detect particular regions of the genome that are under selection (see Vitti et al. 2013). Selection scan methods typically assume that most of the genome is evolving neutrally and that the genome-wide distribution of summary statistics reflects demographic factors. Genomic regions that are outliers from this distribution are presumed to be under selection. In contrast, we are interested in detecting when the genome-wide background is not well-modeled by the Kingman coalescent.

One approach to identifying multiple mergers in genomic data is to use the SFS as a summary statistic. To this end, Birkner et al. (2013) and Blath et al. (2016) and Spence et al. (2016) derived methods for computing the expected SFS of (simultaneous) multiple-merger coalescents. Further, Eldon et al. (2015) showed that it is possible to distinguish between a multiple-merger coalescent of the beta family and the Kingman coalescent with exponential growth using the SFS. In a related approach, Rödelsperger et al. (2014) detected widespread linked selection in the nematode Pristionchus pacificus by demonstrating that the SFS is non-monotonic, a signature of multiple mergers (Neher and Hallatschek 2013; Birkner et al. 2013).

However, existing methods are limited in their ability to distinguish multiple mergers from general models of population-size change. The primary signature of multiple mergers in the SFS is an overabundance of low-frequency mutations relative to the Kingman expectation, which is also the signature of population growth. Eldon et al. (2015) were able to reject exponential growth in favor of multiple mergers, but a more flexible model of growth may be able to fit the multiple-merger SFS (see Myers et al. 2008; Bhaskar and Song 2014). The non-mononotic 
SFS identified by Rödelsperger et al. (2014) is a more robust signature of multiple mergers, but identifying that the SFS increases at high frequencies requires knowing the ancestral allele at each site. Highfrequency mutations are typically much rarer than low-frequency mutations, so misidentifying even a small fraction of ancestral alleles can generate a non-monotonic SFS.

Here, we propose that summary statistics based on the two-site frequency spectrum (2-SFS) - the generalization of the SFS to pairs of nearby sites (Hudson 2001; Ferretti et al. 2018) - are useful for distinguishing between the Kingman coalescent with population growth and multiple-merger coalescents. These statistics may be calculated efficiently from genomic single-nucleotide-variant data. Furthermore, they do not require phasing, recombination maps, or ancestral allele identification and are informative even with small sample sizes. Together, these properties make the 2-SFS useful for demographic modelchecking in a wide range of species.

Following the notation of $\mathrm{Fu}$ (1995), the site frequency spectrum of a sample of $n$ haploid genomes is $\left\{\xi_{i}: 1 \leq i<n\right\}$, where $\xi_{i}$ is the fraction of sites containing a mutation with derived allele count $i$ in the sample. In many cases, the ancestral allele is unknown and so the allele in $i$ samples and the complementary allele in $n-i$ samples are indistinguishable. Therefore, we will mostly consider the folded site frequency spectrum $\left\{\eta_{i}=\xi_{i}+\left(1-\delta_{i, n-i}\right) \xi_{n-i}: 1 \leq i \leq\lfloor n / 2\rfloor\right\}$, where $\delta_{k, k^{\prime}}$ is the Kronecker delta. The SFS and folded SFS can be calculated from a set of single nucleotide polymorphisms (SNPs) without knowing the physical locations of the SNPs.

In contrast, the 2-SFS is a statistic of pairs of sites. We define the 2-SFS, $\left\{\xi_{i j}(d): d>0 ; 1 \leq i, j<n\right\}$, as the fraction of pairs of sites separated by $d$ bases for which there is a mutation with derived allele count $i$ at one site and a second mutation with derived allele count $j$ at the other site. (Note that $\xi_{i j}(d)=\xi_{j i}(d)$ by symmetry.) The 2-SFS has been studied for a non-recombining sites by Ferretti et al. (2018) in a neutral model and by Xie (2011) in a model with selection. We define the folded 2-SFS, $\eta_{i j}(d)$, by analogy to the folded SFS, categorizing pairs of sites by their minor allele frequencies. (For non-recombining sites, the 2-SFS is independent of the distance and so we will suppress the $d$ in our notation.)

In the limit of low per-site mutation rate $(\mu \rightarrow 0)$ and no recombination, all polymorphic sites are bi-allelic and the expected SFS and 2-SFS are related to moments of the genealogical branch length distribution by

$$
\begin{aligned}
\left\langle\xi_{i}\right\rangle & =\mu\left\langle\tau_{i}\right\rangle \\
\left\langle\xi_{i j}\right\rangle & =\mu^{2}\left\langle\tau_{i} \tau_{j}\right\rangle,
\end{aligned}
$$

where $\tau_{i}$ is the total length of branches subtending $i$ leaves of a gene 
genealogy and $\langle\cdot\rangle$ represents the expectation over the distribution of gene genealogies defined by a coalescent model. Thus, the SFS and 2-SFS depend on the distribution of coalescent times as well as the distribution of tree topologies.

$\mathrm{Fu}(1995)$ calculated the first and second moments of the branchlength distribution for non-recombining infinite-sites locus under the standard time-homogeneous Kingman coalescent. He found that $\left\langle\tau_{i} \tau_{j}\right\rangle<$ $\left\langle\tau_{i}\right\rangle\left\langle\tau_{j}\right\rangle$ for all $j \notin\{i,(n-i)\}$. This result, combined with Eq. (1) and (2), implies a negative correlation between mutations at different frequencies: trees generating a mutation with derived allele count $i$ are less likely than average to generate a second mutation with derived allele count $j \notin\{i,(n-i)\}$. (There are positive correlations between mutations at complementary frequencies induced by genealogies whose root node partitions the tree into subtrees of size $i$ and $n-i$.)

Birkner et al. (2013) extended Fu's calculation to a family of multiplemerger coalescents called beta coalescents. This one-parameter family interpolates between the Kingman coalescent and the BolthausenSznitman coalescent as the parameter, $\alpha$, ranges from 2 to 1 . Beta coalescents arise in models with fat-tailed offspring distributions (Schweinsberg 2003; Steinrücken et al. 2013), and the Bolthausen-Sznitman coalescent is the limiting distribution of genealogies in populations that are rapidly adapting or experiencing extensive purifying selection (Neher and Hallatschek 2013). The calculations of Birkner et al. (2013) show positive correlations between $\xi_{i}$ and $\xi_{j}$ for $j \notin\{i, n-i\}$ (Figures 5 and 6 of Birkner et al. 2013). Thus, unlike the standard Kingman coalescent, the beta coalescent can generate positive associations between mutations with different minor allele counts.

In the following, we demonstrate that the positive associations between mutations at different frequencies distinguish the multiplemerger from the Kingman coalescent and that this distinction: (i) applies to Kingman coalescents with time-varying coalescent rates; (ii) is robust to recombination between the sites; (iii) is also a feature of forward-time models with selection, and (iv) can form the basis of a model-checking procedure for demographic inference methods. To demonstrate these four properties, we introduce a transformation of the 2-SFS that we call frequency pointwise mutual information (fPMI). We use a combination of numerical calculations and stochastic simulations to explore the properties of this statistic under different coalescent models. Finally, we demonstrate a graphical model-checking procedure based on fPMI using genomic diversity data from Drosophila melanogaster (Lack et al. 2015). 

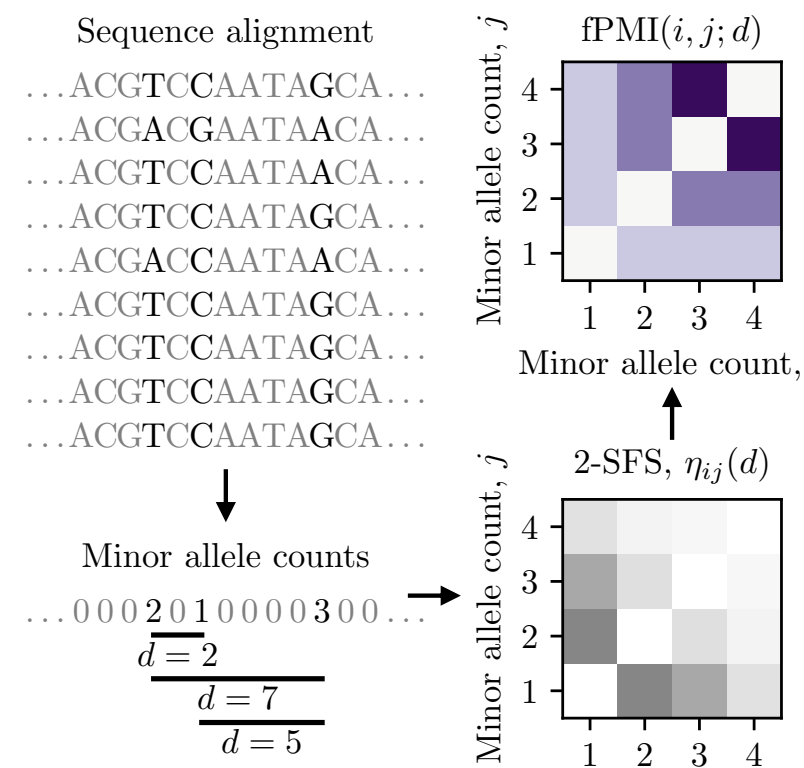

Minor allele count, $i$

$\uparrow$

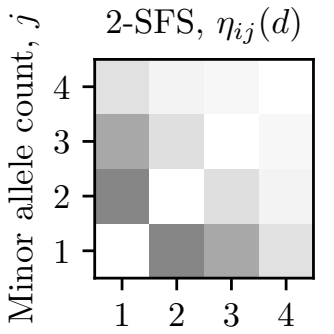

Minor allele count, $i$

Figure 1: Summarizing population genetic data with frequency pointwise mutual information (fPMI). First, a sequence alignment is reduced to the minor allele frequencies and positions of segregating mutations. Then, for a given distance, $d$, the 2-SFS gives the number of pairs of sites with each combination of minor allele frequencies. Finally, fPMI is calculated from the 2-SFS according to Eq. (3).

\section{Methods}

\section{Frequency pointwise mutual information}

We aim to quantify the dependence between minor allele counts at a pair of sites, particularly for sites with different counts $(i \neq j)$. To do so, we define the frequency pointwise mutual information as

$$
\operatorname{fPMI}(i, j ; d)=\log \frac{\left\langle\eta_{i, j}(d)\right\rangle}{\left\langle\eta_{i}\right\rangle\left\langle\eta_{j}\right\rangle}
$$

Figure 1 shows the steps to compute fPMI from a sample of sequences.

In information theory, the pointwise mutual information (PMI) of a pair of random variables, $X$ and $Y$, is a transformation of the prob- 
ability mass function, $p(x, y)$, given by

$$
\operatorname{PMI}(x, y)=\log \frac{p(x, y)}{p(x) p(y)}=\log \frac{p(x \mid y)}{p(x)}
$$

where $p(x)=\sum_{y} p(x, y)$ and similarly for $p(y)$ (Church and Hanks 1990). PMI measures the change in the probability that $X=x$ given knowledge that $Y=y$. When $X$ and $Y$ are independent, $\operatorname{PMI}(x, y)=0$ for all $x, y$. When $X$ and $Y$ are not independent, $\operatorname{PMI}(x, y)>0$ implies that $p(x \mid y)>p(x)$, and $\operatorname{PMI}(x, y)<0$ implies the converse. The expectation of PMI over the joint distribution of $X$ and $Y$ is known as the mutual information of $X$ and $Y$ (Cover and Thomas 1991).

We can interpret the 2-SFS as a joint probability mass function over minor allele counts. Given a coalescent model, the minor allele count at an arbitrary site is a random variable over $\{0, \ldots,\lfloor n / 2\rfloor\}$ with probability mass function $p(i)=\left\langle\eta_{i}\right\rangle$, where we define $\eta_{0}$ to be the fraction of monomorphic sites. Similarly, the minor allele counts at two sites separated by $d$ bases are random variables with joint probability mass function $p_{d}(i, j)=\left\langle\eta_{i j}(d)\right\rangle$. Comparing, Eq. (3) with Eq. (4) shows that $\mathrm{PPMI}$ is the standard pointwise mutual information for pairs of minor allele counts.

This transformation of the 2-SFS has several useful properties. First, because fPMI is based on the minor allele frequencies, we may compute it without knowing the ancestral allele. Second, the results of $\mathrm{Fu}(1995)$ for the folded SFS imply that $\mathrm{fPMI}(i, j)<0$ for $i \neq j$ for non-recombining sites under the time-homogeneous Kingman coalescent. Finally, fPMI normalizes for distortions in the coalescence time distribution reflected in the single-site SFS. In the Kingman coalescent, population-size variation alters the coalescence time distribution but preserves the distribution of tree topologies. We will show that, because fPMI normalizes the 2-SFS by the product of single-site spectra, it is largely insensitive to the distribution of coalescence times. Thus, fPMI primarily reflects the distribution of topologies, which distinguishes the multiple-merger coalescent from the Kingman coalescent.

Furthermore, the same normalization renders fPMI insensitive to ascertainment bias. Variant detection methods typically compare sampled sequences to a reference sequence and "call" a variant site when there is sufficient evidence that at least one sample differs from the reference at that site. As a result, the ascertainment probability, $q_{i}$, of a mutation is an increasing function of its true allele count $i$, skewing the expected ascertained SFS $\left(\left\langle\eta_{i}\right\rangle_{\text {asc }}=\mu\left\langle\tau_{i}\right\rangle q_{i}\right)$ toward high-frequency mutations. However, provided that variant detection at each site is approximately independent, the primary effect of ascertainment bias in Eq. (3) is to multiply the numerator and denominator by a common factor of $q_{i} q_{j}$. Because these factors cancel, fPMI is less sensitive than the raw SFS and 2-SFS to ascertainment effects. 


\section{Binned allele frequencies}

With finite data, estimates of the 2-SFS will be noisy. This is particularly true for $i, j \gg 1$ because $\left\langle\eta_{i j}\right\rangle$ decays like $(i j)^{-1}$ for the standard Kingman coalescent (Fu 1995) and faster than this in models with population size growth or multiple mergers. We show in Results that a positive association between mutations with high minor allele counts and mutations with low minor allele counts is a signature of multiple mergers. Thus, to detect multiple mergers, we use a binned form of the SFS and 2-SFS:

$$
\begin{aligned}
\eta_{\mathrm{lo}}\left(i_{c}\right) & =\sum_{i=1}^{i_{c}-1} \eta_{i} \\
\eta_{\mathrm{hi}}\left(i_{c}\right) & =\sum_{i=i_{c}}^{\lfloor n / 2\rfloor} \eta_{i} \\
\eta_{\mathrm{hi}, \mathrm{lo}}\left(d ; i_{c}\right) & =\sum_{i=1}^{i_{c}-1} \sum_{j=i_{c}}^{\lfloor n / 2\rfloor} \eta_{i j}(d),
\end{aligned}
$$

where $i_{c}$ is an arbitrary cutoff between high and low minor allele frequency. Binning provides a stable estimate of the 2-SFS for large sample sizes because we may adjust $i_{c}$ to ensure a large number of sites in both the high-minor allele count and low-minor allele count bins. We note that a less coarse-grained binning scheme may strike a balance between sampling noise and preserving more detailed information about allele frequencies.

We compute the pointwise mutual information of the binned distribution as

$$
\operatorname{hiloPMI}\left(d ; i_{c}\right)=\log \frac{\left\langle\eta_{\mathrm{hi}, \mathrm{lo}}\left(d ; i_{c}\right)\right\rangle}{\left\langle\eta_{\mathrm{lo}}\left(i_{c}\right)\right\rangle\left\langle\eta_{\mathrm{hi}}\left(i_{c}\right)\right\rangle} .
$$

While we will focus on hiloPMI, one could similarly calculate five other pointwise mutual information statistics from the binned 2-SFS (e.g., the PMI between monomorphic sites and sites with high minor allele counts).

\section{Weighted fPMI}

For plotting purposes, we use a weighted version of the fPMI:

$$
\operatorname{wfPMI}(i, j: d)=\frac{\left\langle\eta_{i j}\right\rangle}{\mu^{2}\left\langle T_{2}\right\rangle^{2}} \operatorname{fPMI}(i, j ; d),
$$

where $T_{2}$ is the coalescence time for a sample of size two. The numerator of the weighting factor in Eq. (9) emphasizes the most common 
pairs of minor allele counts. The denominator, which is proportional to the square of the expected pairwise diversity, $\Pi$, ensures that wfPMI is invariant to changes in the mutation rate and average pairwise coalescence time.

\section{Computing branch-length moments}

We implemented numerical computations of the moments of the branch lengths $\left\langle\tau_{i}\right\rangle$ and $\left\langle\tau_{i j}\right\rangle$. For the Kingman coalescent with time-varying coalescent rate, we used equations (1)-(12) of Živković and Wiehe (2008). For the beta coalescent, we implemented the recursion described by Birkner et al. (2013).

Functions for computing the branch-length moments are implemented in python and available in the git repository for this project (https://github.com/dp-rice/multiplemergers). Our Kingman coalescent code can compute moments for exponentially growing and two-epoch piecewise constant models, but could be extended to allow for other models. The formulas of Živković and Wiehe (2008) exhibit numerical instability related to the instability described in Griffiths and Tavaré (1994). Thus, these formulas are only practical for sample sizes up to $n \approx 40$. The recursion of Birkner et al. (2013) is $\mathcal{O}\left(n^{4}\right)$ and is also only practical for samples up to $n \approx 50$.

\section{Coalescent simulations}

We ran coalescent simulations using a custom version of msprime (Kelleher et al. 2016) capable of multiple mergers, based on modifications made by Joe Zhu (https ://github.com/shajoezhu). This code, together with python wrapper scripts and utility functions to run simulations and calculate the 2-SFS and fPMI from the msprime output is available at https://github.com/dp-rice/multiplemergers.

For each coalescent model, we simulated at least $10^{4}$ independent infinite-sites loci, each with length $d$ and per-basepair recombination rate $r$ resulting in a per-locus total recombination rate $d r$. (See SI for parameter combinations.) We chose values of $d r$ so that the realized values of $d r\left\langle T_{2}\right\rangle$ varied over several orders of magnitude. For each locus, we measured $\left\{\tau_{i}: i=1, \ldots, n-1\right\}$ in the two genealogies at the ends of the locus. We then calculated the expectations $\left\{\left\langle\tau_{i}\right\rangle\right\}$ and $\left\{\left\langle\tau_{i j}\right\rangle\right\}$ by averaging over independent loci. These expectations allow us to calculate all of the 2-SFS statistics defined above.

\section{Forward-time simulations of selective sweeps}

We simulated a model of recurring selective sweeps using the software SLiM (Messer 2013). In all of these simulations, we simulated a popula- 
tion of 500 diploids for $10^{4}$ generations. Each haploid genome consisted of a single genomic element $L=10^{8}$ basepairs long with recombination rate per-basepair $r=10^{-8}$ and per-basepair mutation rate $\mu=10^{-7}$. We simulated two types of mutations: neutral mutations and beneficial mutations with additive effects and selection coefficient $s=0.1$. With these parameters, $2 N s=100$, so beneficial mutations are strongly selected and will sweep in $T_{\text {sweep }} \sim s^{-1} \log N s \approx 50$ generations. Such sweeps will affect a region of the chromosome $d_{\text {sweep }} \sim\left(r T_{\text {sweep }}\right)^{-1} \approx$ $2 \times 10^{6}$ basepairs long. Thus $d_{\text {sweep }} \ll L$, which will minimize edge effects of simulating a finite chromosome.

In order to vary the effects of sweeps on neutral diversity, we varied the fraction of mutations that are beneficial, $f_{\text {sel }}$, over several orders of magnitude: $f_{\text {sel }} \in\left\{10^{-6}, 10^{-5}, 10^{-4}, 10^{-3}\right\}$. For each $f_{\text {sel }}$, we ran 100 independent replicate simulations and computed $\eta_{i}$ and $\eta_{i j}$ of neutral mutations averaged over all replicates.

SLiM parameter files, python wrapper scripts for parsing output, and snakemake files for running simulations are available at https: //github.com/dp-rice/multiplemergers.

\section{Analysis of D. melanogaster data}

We analyzed sequence data from the DPGP3 data set, which consists of haploid consensus sequences from $\sim 200$ flies, obtained via the haploid embryo method of Langley et al. (2011). The SNP calls that characterize these sequences were subjected to a variety of quality filters described in Lack et al. (2015). We obtained the DPGP3 consensus sequence files version 1.1 from www. johnpool. net/genomes.html. These files contain the sequence alignments of all flies in the sample on all chromosome arms. We also downloaded the Nov. 3, 2016 spreadsheet of inversions available at the link above. For each chromosome arm, we excluded any samples with an inversion in that arm and then down-sampled to $n=100$ by selecting the first 100 remaining samples in alphanumeric order by sample name. As a result, the data for each chromosome arm is from a slightly different subset of the individuals.

We calculated the average pairwise diversity, $\Pi$, as a function of position for each autosomal chromosome arm (Fig. S1). Pairwise diversity is high in the middle of each chromosome arm and lower near the centromeres and telomeres, in agreement with calculations by CorbettDetig et al. (2015). Our modeling - and coalescent-based demographic inference in general - assumes that the distribution of gene genealogies is homogeneous along the chromosome. Therefore, we selected a 13-16 Mb "central" region of each arm with relatively homogeneous values of $\Pi$ for further analysis. The boundary positions of these central regions are given in Table S1.

In order to ensure that the segregating mutations reflect true ge- 
netic diversity and not variation in calling errors, we excluded sites with fewer than 90 of the 100 genotypes called. This leaves over $90 \%$ of all sites and does not substantially alter the fraction of polymorphic sites (Table S2).

We fit a demographic model to the folded SFS of fourfold degenerate sites for each chromosome arm separately using fastNeutrino (Bhaskar et al. 2015). Following Ragsdale and Gutenkunst (2017) we fit a three-epoch piecewise constant $-N$ model:

$$
N(t)= \begin{cases}N_{1} & 0 \leq t \leq t_{1} \\ N_{2} & t_{1}<t \leq t_{2} \\ N_{\mathrm{anc}} & t>t_{2}\end{cases}
$$

estimating both change-points $\left(t_{1}<t_{2}\right)$ and population sizes $\left(N_{1}, N_{2}\right)$. We specified the ancient population size $N_{\text {anc }}=3 \times 10^{5}$, as in Ragsdale and Gutenkunst (2017). For all four chromosomes, fastNeutrino inferred similar population growth. Fitted parameters are presented in Table S3. We simulated the SFS and 2-SFS under the fitted parameters using msprime.

In addition to the average SFS used to fit the model, we computed the average 2-SFS for pairs of sites at distances between 3 bp and $5 \mathrm{~Kb}$. Because we are using fourfold degenerate sites, we only computed the 2-SFS for distances that are multiples of three basepairs. For comparison between data and simulations, we scale the distances in basepairs by a critical distance $d_{c}=\left(r\left\langle T_{2}\right\rangle\right)^{-1}$. We used a genome-wide recombination rate of $r=2 \times 10^{-8}$ per-basepair per generation (Comeron et al. 2012). We estimated $\left\langle T_{2}\right\rangle$ by $\Pi / 2 \mu$. For $\Pi$, we used the average pairwise diversity at fourfold degenerate sites in the central region of each chromosome arm. For $\mu$, we used a genome-wide mutation rate of $3 \times 10^{-9}$ per-basepair per-generation (Keightley et al. 2014). These estimates are not precise, but only serve to scale genetic distances to the correct order of magnitude.

A snakemake pipeline, python scripts, and jupyter notebooks to replicate our data processing, model fitting, simulations, and analysis of the DPGP3 data are available at https://github.com/dp-rice/ multiplemergers.

\section{Results}

\section{Population growth versus the beta coalescent in non- recombining loci}

We first compared the PPMI of the Kingman coalescent with and without population growth to the fPMI of the beta coalescent, for pairs of 


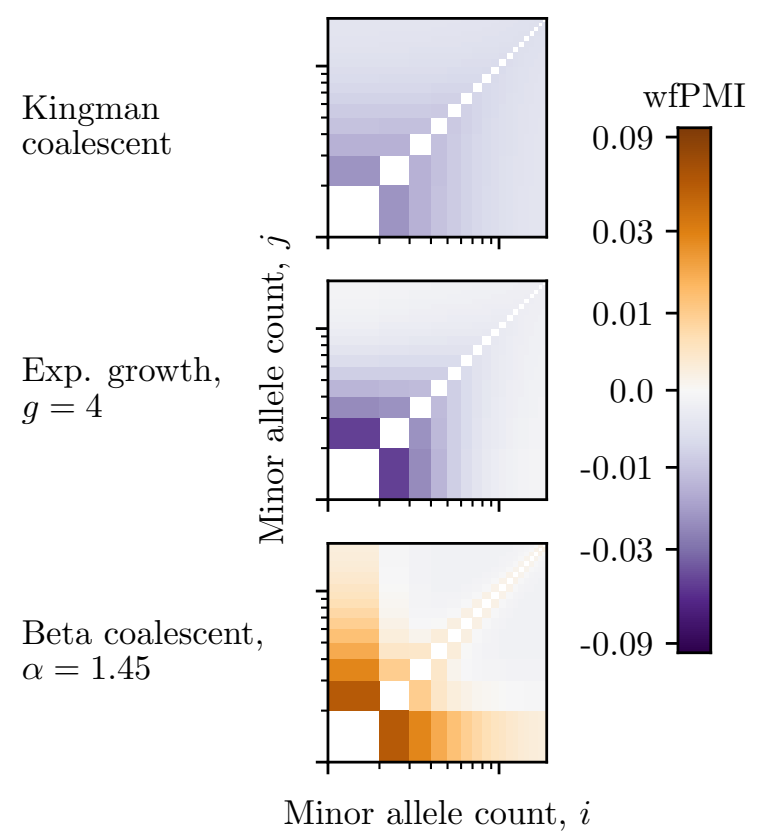

Figure 2: Weighted frequency pointwise mutual information for three coalescent models: the time-homogeneous Kingman coalescent, the Kingman coalescent with exponential growth $(g=4)$, and the beta coalescent $(\alpha=1.45)$. For all models $n=39$. Growth and $\alpha$ parameters were chosen to generate average SFS with similar Tajima's D. The diagonal, $i=j$, is masked.

sites without recombination. We are interested in whether fPMI can distinguish beta from Kingman coalescent models that produce similar distortions in the SFS. To this end, we computed a version of Tajima's D (Tajima 1989) normalized to be invariant to changes in the average pairwise coalescence time: $D=\left(\Pi-\hat{\theta}_{W}\right) / \Pi$, where $\hat{\theta}_{W}$ is Watterson's theta (Watterson 1975). Negative values of $D$ indicate an overabundance of low-frequency mutations relative to the time-homogeneous Kingman expectation. All results are computed numerically using the results of Fu (1995), Živković and Wiehe (2008), and Birkner et al. (2013) (see Methods).

As a first example, Fig. 2 shows the wfPMI for the constant- $N$ Kingman coalescent; the Kingman coalescent with exponential growth, $N(t)=N_{0} \exp \left(-g \frac{t}{N_{0}}\right)$; and a beta coalescent intermediate between the Kingman and Bolthausen-Sznitman coalescents. Exponential growth with $g=4$ and the beta coalescent with $\alpha=1.45$ both generate 


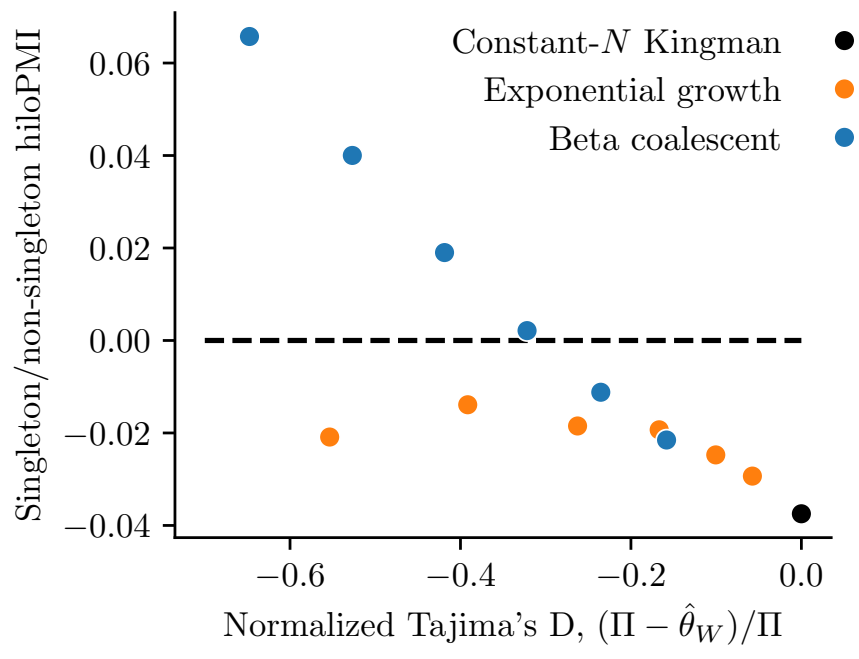

Figure 3: Pointwise mutual information between singletons and nonsingletons for Kingman coalescents with exponential growth and beta coalescents. The exponential growth rate, $g$, ranges from 0.25 to 8.0 in coalescent time units. The beta coalescent parameter, $\alpha$, ranges from 1.75 (nearly Kingman) to 1.25 (nearly Bolthausen-Sznitman).

similar substantial distortions in the SFS, $D=-0.4$. However, exponential growth does not qualitatively change the fPMI. In particular, $\mathrm{fPMI}_{i, j}<0$ for all $i \neq j$, as in the constant- $N$ Kingman coalescent. On the other hand, the beta coalescent generates positive fPMI. The effect of multiple mergers is strongest on fPMI between high and low minor allele counts. This justifies binning the SFS and 2-SFS into high- and low-minor allele count bins as defined above.

To generalize this finding, we computed the binned hiloPMI between singletons and non-singletons $\left(i_{c}=1\right)$ for a range of $g$ and $\alpha$. Figure 3 shows that as the population growth rate increases, distorting the SFS, hiloPMI increases relative to the constant- $N$ Kingman, but remains negative. On the other hand, beta coalescents that generate similar distortions in the SFS, generate larger changes in hiloPMI, including positive values. Thus, the 2-SFS, and hiloPMI in particular, are capable of capturing the effects of multiple mergers beyond the distortions in branch lengths. 


\section{Pointwise mutual information between recombining sites}

We have shown in the previous section that fPMI between pairs of non-recombining sites can discriminate between the Kingman coalescent with population growth and the beta coalescent. However, most demographic inference is performed on regions of the genome with non-zero recombination rates. Therefore, it is important to assess the robustness of our approach to recombination between sites.

To measure the effect of recombination on fPMI, we ran coalescent simulations using a version of the program msprime (Kelleher et al. 2016) modified to allow for multiple mergers (See Methods). In particular, we simulated a model where the marginal trees are generated by the beta coalescent process, as in our numerical calculations above. In this model, marginal genealogies will follow the beta coalescent distribution, and the average SFS will be given by the formula in Birkner et al. 2013. We also simulated data from two models of population growth: exponential growth and a two-epoch piecewise-constant model.

Figure 4 shows the weighted fPMI for three genetic distances between sites in a constant- $N$ Kingman coalescent, a Kingman coalescent with exponential growth, and a beta coalescent. As in the nonrecombining case, the constant- $N$ and exponential-growth Kingman models have similar fPMI. In both models, fPMI $>0$ for $i \approx j$ for $d r\left\langle T_{2}\right\rangle \sim 1$. This is presumably because trees at nearby sites contain clades with similar numbers of leaves. These positive correlations do not extend to $i \gg j$, which is the signal of multiple mergers in our binned hiloPMI. On the other hand, the positive fPMI in the beta coalescent persists for $d r\left\langle T_{2}\right\rangle>1$. Thus, the signal of multiple mergers in $\mathrm{PPMI}$ is robust to recombination.

Figure 5 shows hiloPMI $\left(d, i_{c}\right)$ in both models of growth and the beta coalescent, for a range of $d r\left\langle T_{2}\right\rangle$ and three different choices of $i_{c}$. Each curve represents a particular parameter combination and coalescent model and is colored by the distortion in the average SFS (Tajima's D), which is independent of the recombination rate. At low recombination rates, hiloPMI may be greater in growing populations than in constant- $N$ populations (dotted lines), but is always less than zero, consistent with the results for non-recombining sites. When $d r\left\langle T_{2}\right\rangle \geq 1$, hiloPMI may be slightly positive for large $i_{c}$, but is smaller in growing populations than in the constant- $N$ Kingman model. In all Kingman models, hiloPMI decays to zero for $d r\left\langle T_{2}\right\rangle \gg 1$.

In contrast, the beta coalescent generates hiloPMI that is consistently greater than the constant- $N$ Kingman. As with non-recombining sites, the hiloPMI is also greater in the beta coalescent model than in models of population growth that generate similar distortions in the 


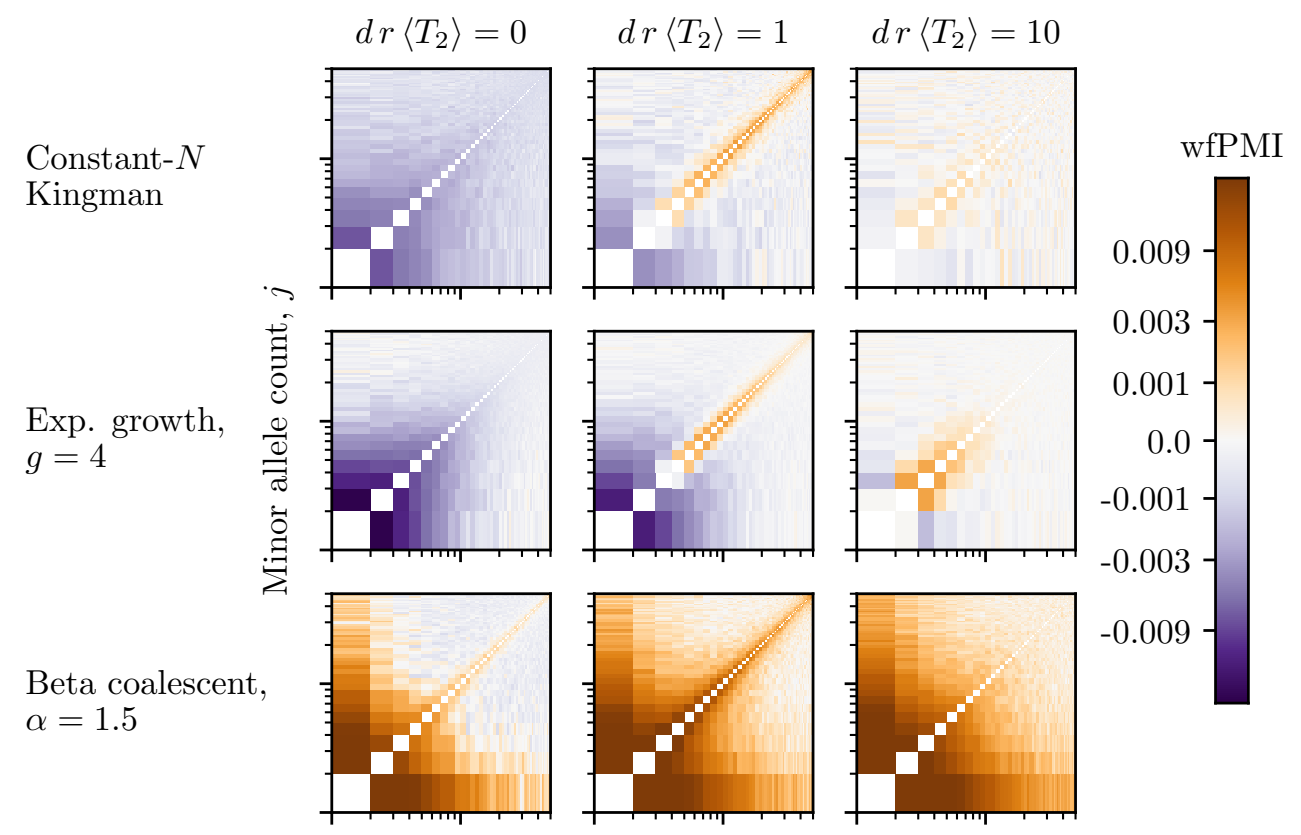

Minor allele count, $i$

Figure 4: Weighted frequency pointwise information for three recombination rates with sample size $n=100$. As in Figure 2, the diagonal elements are masked, and the growth and multiple-merger parameters were chosen to generate average SFS with similar Tajima's D. 


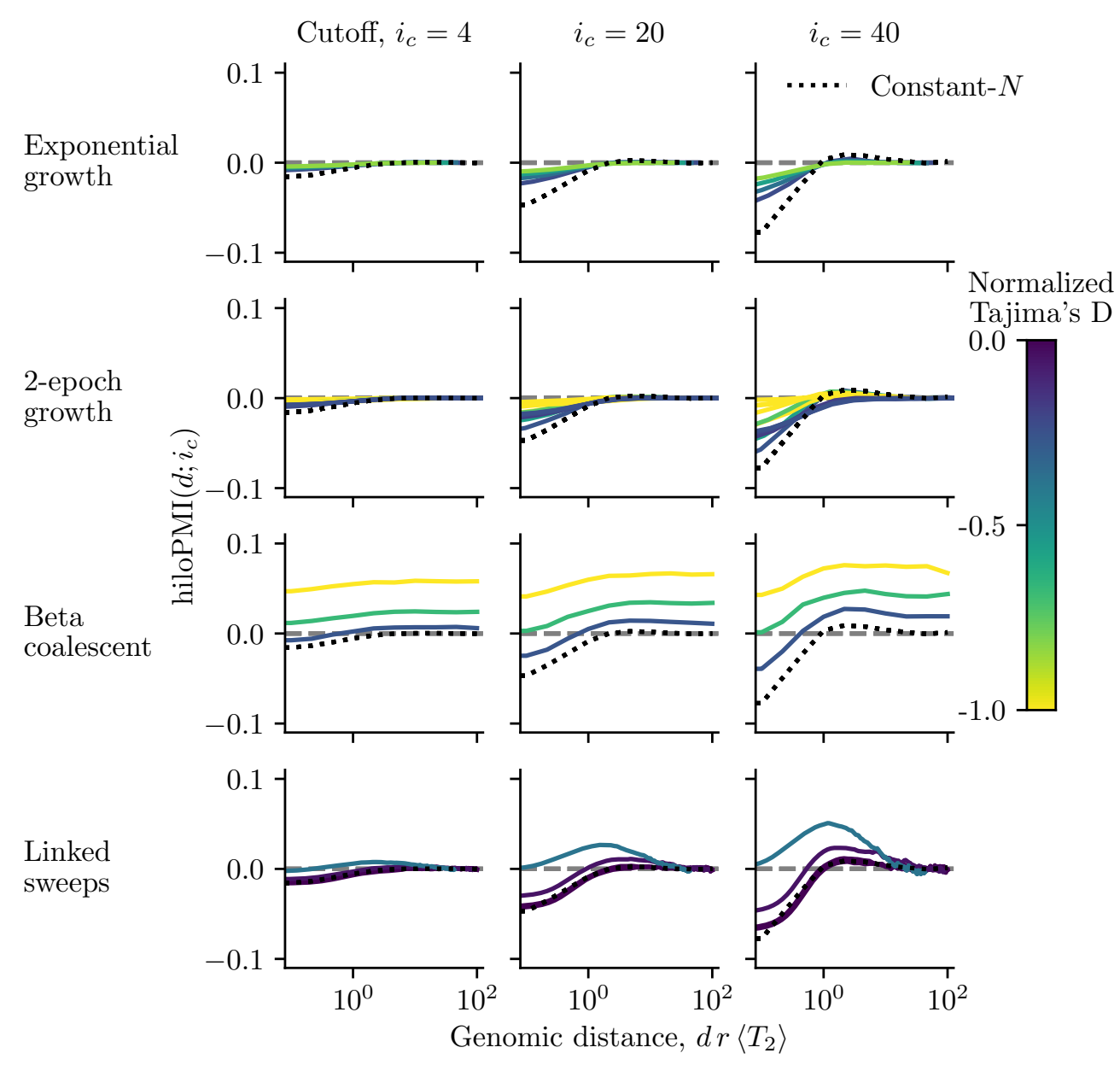

Figure 5: Pointwise mutual information between high- and low-minor allele count mutations (hiloPMI) versus genetic distance. Each curve represents a different combination of coalescent parameters. The first three rows show coalescent simulations in msprime. Exponential growth rate $g$ varies logarithmically from 1 to 8.0 in coalescent time units. Piecewise constant change-points range from 0.01 to 1 in coalescent time units, and the ratio of ancestral to modern population size ranges from 0.01 to 0.2 . The fourth row shows forward-time SLiM simulations with selective sweeps at linked sites (beneficial mutation fraction $f_{\text {sel }} \in\left\{10^{-6}, 10^{-5}, 10^{-4}, 10^{-3}\right\}$, see Methods). Lines are colored by the distortion in the SFS, as measured by Tajima's D. The dashed line shows the constant- $N$ Kingman coalescent. 


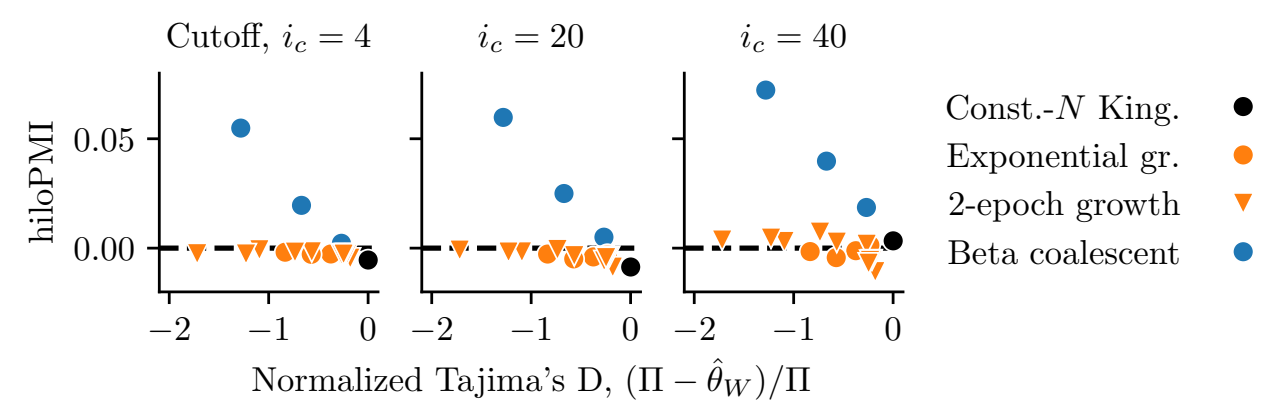

Figure 6: Pointwise mutual information between high- and low-minor allele count mutations (hiloPMI) versus normalized Tajima's D for simulations with recombination. All values are for $d r\left\langle T_{2}\right\rangle \sim 1$ and $n=100$. Orange points show Kingman coalescent simulations with population growth. Blue points show beta coalescent simulations.

SFS. This is true across recombination rates and high/low cutoff minor allele counts.

These results demonstrate that hiloPMI is capable of discriminating between coalescent models even when there is recombination between sites. In particular, population growth has very little effect on hiloPMI, especially for $d r\left\langle T_{2}\right\rangle \gtrsim 1$, while multiple mergers increase hiloPMI across the range of $d r\left\langle T_{2}\right\rangle$. Figure 5 also suggests that the most informative genomic distance for distinguishing multiple mergers from population growth is $d r\left\langle T_{2}\right\rangle \sim 1$. In a later section, we will demonstrate how to use these results to implement a model checking procedure based on plotting hiloPMI as a function of genomic distance.

Figure 6 highlights the relative invariance of hiloPMI at intermediate genomic distances to population growth. While population growth in a Kingman coalescent model can strongly distort the SFS, as measured by Tajima's D, it has very little effect on the hiloPMI for sites $d r\left\langle T_{2}\right\rangle \sim 1$ apart. This holds for exponential as well as piecewise constant growth. In contrast, the beta coalescent induces large positive hiloPMI for the same values of Tajima's D. The figure also shows that this result is robust to the choice of cutoff minor allele count for binning.

There is one other salient feature of Fig. 5: hiloPMI does not decay to zero at long distances in the beta coalescent. This behavior relates to the results of Eldon and Wakeley (2006), who showed that a model with "jackpot" reproductive events can generate infinite-range linkage disequilibrium. Further, Eldon and Wakeley (2006) showed that different scalings of rates of mutation, pairwise coalescence, multiple- 
merger coalescence, and recombination lead to different behaviors of diversity and linkage disequilibrium. Our implementation of the beta coalescent model with recombination corresponds to a particular scaling limit where recombination does not have time to decorrelate trees during multiple-merger events, even at infinite genetic distances. We do not expect this behavior to be universal in multiple-merger coalescents with recombination.

\section{Linked selective sweeps}

Various authors have shown that natural selection can generate multiplemerger coalescents at linked neutral sites (e.g., Durrett and Schweinsberg 2005; Coop and Ralph 2012; Neher and Hallatschek 2013; Desai et al. 2013; Seger et al. 2010). However, our simulations of the beta coalescent with recombination are of an explicitly neutral model. Thus, they are at best an approximation to the selective models cited above. It is therefore important to verify that selection at linked sites can, in fact, generate the sorts of signals in fPMI that we have detected in the beta coalescent.

To test this proposition, we performed forward-time simulations of recurring selective sweeps using the software SLiM (Messer 2013). In these simulations, we simulated individual chromosomes with homogenous recombination and two types of mutations: neutral and beneficial with a fixed selection coefficient, $s$. Both types of mutations occurred at random, uniformly distributed along the chromosome. By varying the recombination rate, neutral and beneficial mutation rates, population size, and selection coefficient, we varied the rate of selective sweeps linked to neutral sites.

The bottom row of Fig. 5 shows the results of these simulations. For intermediate genetic distances, $d r\left\langle T_{2}\right\rangle \sim 1$, the effects of linked sweeps are qualitatively similar to the effects of the beta coalescent. That is, when sweeps are sufficiently frequent to distort the SFS, as measured by Tajima's D, they also increase hiloPMI. The primary difference between the forward-time sweeps and beta coalescent simulations is that the distortions caused by sweeps decay to zero for $d r\left\langle T_{2}\right\rangle \gg 1$. This decay is expected because the effect of a single selective sweep on the genealogy is localized around the position of beneficial mutation.

\section{Application to Drosophila melanogaster: Coales- cent model checking}

Our results above show that fPMI and its binned analog, hiloPMI, are useful for distinguishing population growth from the effects of multiple mergers, even when population growth generates similar distortions in 
the SFS. We therefore propose the following model-checking procedure for demographic inference methods:

(i) Fit a demographic model to data.

(ii) Simulate genealogies under the fitted model (using msprime or other coalescent simulator). Calculate the $\operatorname{PPMI}(d)$ and hiloPMI $\left(d ; i_{c}\right)$ predicted by the model.

(iii) Calculate $\operatorname{fPMI}(d)$ and hiloPMI $\left(d ; i_{c}\right)$ from the data.

(iv) Compare true to predicted statistics to evaluate model fit.

This procedure checks whether the demographic model is consistent with a feature of the data that was not used in fitting the model. Inconsistency suggests that the inferred $N(t)$ may be an artifact of natural selection, skewed offspring distributions, etc., rather than reflecting the true historical population size.

In this section, we illustrate the procedure outlined above by using genomic diversity data from the Drosophila melanogaster DGPG3 panel (Lack et al. 2015). The DPGP3 data consists of haploid consensus sequences from $\sim 200$ wild-caught flies from a Zambian population known to be mostly free of cosmopolitan admixture. Recently, several groups have used the DPGP3 data to estimate the populationsize history of D. melanogaster (Terhorst et al. 2017; Ragsdale and Gutenkunst 2017). On the other hand, it is widely believed that the genetic diversity of Drosophila is strongly shaped by natural selection (e.g., Elyashiv et al. 2016; Garud and Petrov 2016). Thus, this data is a good candidate for demonstrating the utility of fPMI for assessing coalescent model fit.

After filtering for missing genotypes, removing chromosome arms with known inversions, downsampling to $n=100$ samples per autosomal chromosome arm, and identifying 4-fold degenerate sites, we selected the central region of each chromosome characterized by consistent high diversity (Methods). Because the average pairwise diversity varies between arms - possibly reflecting selection or different sets of segregating inversions - we performed all subsequent calculations on each arm independently. We fit a demographic model to the site frequency spectra of these central regions using fastNeutrino (Bhaskar et al. 2015). We fit a 3 -epoch piecewise-constant model, with four free parameters: two changepoints and two population size ratios. We report our fitted parameters in Table S3. We then simulated under our fitted model using msprime and computed the expected and observed SFS, fPMI, and hiloPMI (Fig. 7, Methods).

The first row of Fig. 7 shows that the expected SFS under the fit demographic models agree with the observed SFS, demonstrating that a time-varying $N(t)$ can explain this aspect of the data well. In contrast, the second row shows the expected and observed weighted fPMI 

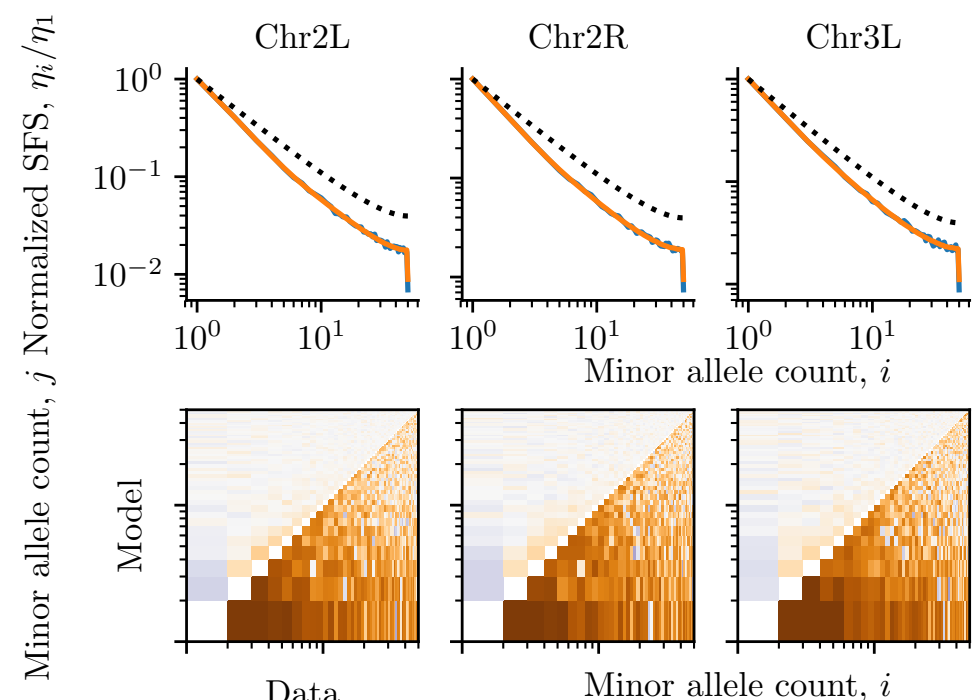

Minor allele count, $i$
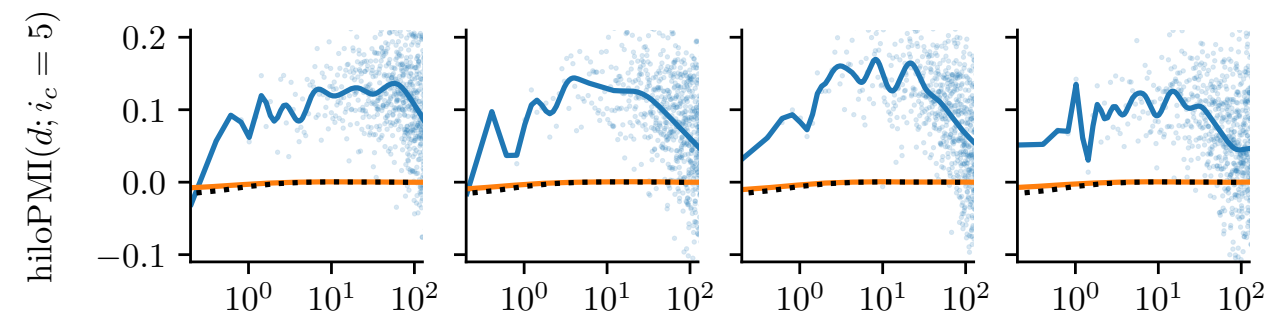

Genomic distance, $d r\left\langle T_{2}\right\rangle$

Figure 7: DPGP3 data and coalescent model predictions. First row: observed (blue) and expected (orange) site frequency spectrum compared to constant- $N$ Kingman coalescent (dotted lines). Second row: Expected (upper triangle) and observed (lower triangle) weighted fPMI averaged over all pairs of sites less than $15 d_{c}$ apart. Third row: Observed (blue) and expected (orange) hiloPMI versus genetic distance. Cutoff minor allele count, $i_{c},=5$. Solid blue curves show cubic spline fit. Dotted lines show the expectation under the constant- $N$ Kingman coalescent. 


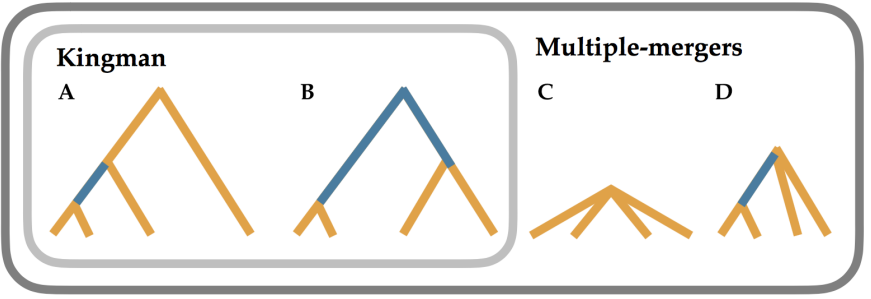

Figure 8: Genealogies with a sample size of $n=4$. Opportunities for singleton/tripleton mutations are in orange. Opportunities for doubletons are in blue. (Note: there is a third possible multiple-merger topology, not shown.)

averaged over distances less than 200 bp apart, which corresponds to to $d / d_{c}<15$. Here, the data shows strong positive associations between nearby alleles at different frequencies, while the model of population growth predicts weak negative associations except adjacent to the diagonal. This pattern extends across a range of genomic distances, $d / d_{c} \in\left(10^{-1}, 10^{2}\right)$ (Fig. 7 , third row). As a result, we may conclude that the data is not well explained by the Kingman coalescent with population growth.

Note that the hiloPMI decays toward zero at large distances, matching the expectation from simulations with selective sweeps rather than the beta coalescent. However, we caution against concluding that sweeps are necessarily responsible for the deviations from the Kingman expectation.

\section{Discussion}

We have shown that fPMI and its binned analog hiloPMI are sensitive to multiple mergers, but largely invariant to population growth in the Kingman coalescent. These properties make them well-suited for coalescent model checking. We demonstrated a model-checking procedure on data from $D$. melanogaster, which is believed to be strongly shaped by natural selection, and found evidence that population growth alone cannot explain the positive associations between high and low frequency mutations.

We can get an intuitive understanding for why fPMI distinguishes among coalescent models by considering a sample of four chromosomes. In the Kingman coalescent, there are only two possible tree topologies (Figure 8). Furthermore, the total branch length is independent of the topology (Wakeley 2009). As a result, there is a trade-off between the length of branches leading to singleton/tripleton mutations on one 


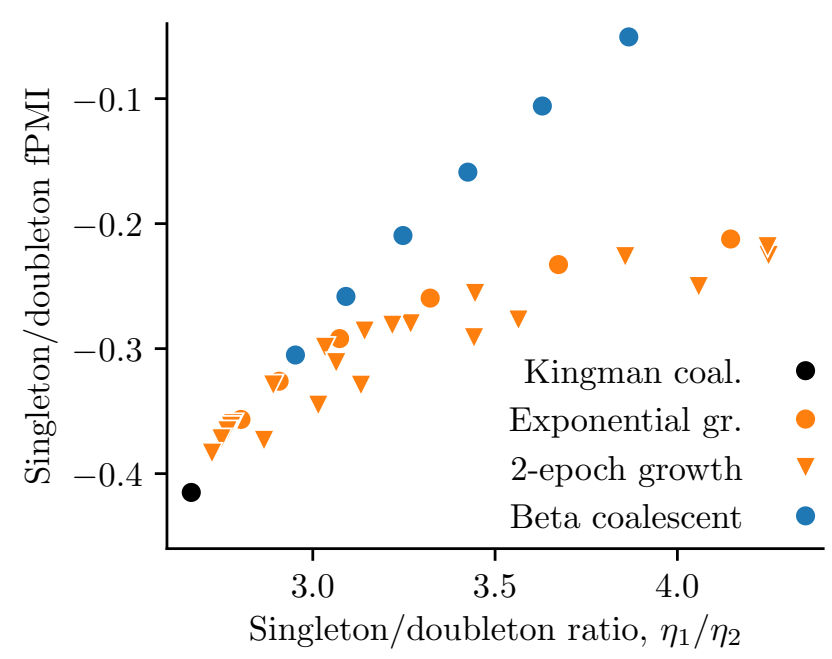

Figure 9: Distortions in fPMI vs. distortions in the SFS for $n=4$. Parameters for beta coalescents and exponential growth are as in Fig. 3. For the piecewise-constant- $N$ model, the fold-change in $N$ varies from 2 to $2^{4}$ and the time of this change varies from $2^{-4}$ to 2 in coalescent time units.

hand, and the branch length leading to doubletons on the other. Genealogies with topology (A) will have more opportunities for the former and loci with topology (B) will have more opportunities for the latter. Conditional on observing a doubleton at a site, it is thus more likely that the genealogy has topology (B) and so the expected number of singletons at sites with the same genealogy is lower than average. In terms of the 2-SFS, we have $\left\langle\eta_{12}\right\rangle<\left\langle\eta_{1}\right\rangle\left\langle\eta_{2}\right\rangle$.

On the other hand, multiple mergers induce correlations between the tree topology and the total branch length. For example, topology (C) has less opportunity for singletons and less opportunity for doubletons than (A) or (B), even though the expected proportion of singletons is higher. Thus, observing any mutation at all makes topology (C) less likely and the expected number of other mutations at all frequencies higher. If multiple-merger events are frequent enough, this effect may dominate the tradeoff between (A) and (B) so that $\left\langle\eta_{12}\right\rangle>\left\langle\eta_{1}\right\rangle\left\langle\eta_{2}\right\rangle$.

As argued above, $\left\langle\eta_{12}\right\rangle$ is also distorted by changes in the coalescent time distribution induced by population growth. Figure 9 demonstrates that fPMI accounts for this fact by normalizing by the SFS. Figure 9 plots fPMI $(1,2)$ against the ratio of singletons to doubletons $\eta_{1} / \eta_{2}$ for the beta coalescent and two models of population growth: exponential growth and a piecewise-constant model with two epochs. 
In the latter model, we vary both the fold-change in $N$ and the time of the change. Like Tajima's D, the singleton/doubleton ratio measures distortion in the SFS relative to the constant- $N$ Kingman coalescent. (In fact, with $n=4$, this ratio captures all of the distortion in the SFS.) As with the larger sample size (Fig. 3), multiple mergers generate larger distortions in fPMI than population growth does, accounting for the distortions in the SFS. Moreover, the results for two different models of growth coincide, suggesting that the functional form of the population growth does not strongly influence fPMI.

We focus here on demographic inference methods that explicitly use the Kingman coalescent model for calculations. However, another popular class of methods are based on forward-time models, such as the Wright-Fisher model (e.g., Gutenkunst et al. 2009; Sheehan et al. 2013). We believe that fPMI is useful for model-checking with these methods as well. This is because forward-time neutral models each have an associated dual coalescent model (Etheridge 2011), which can be used to compute the expected fPMI as outlined in this paper. Furthermore, non-neutral models also generate predictions about the 2-SFS and thus fPMI. In principle, any fitted model that allows calculation or simulation of the 2-SFS may be checked using fPMI. However, the suitability of fPMI for discriminating among arbitrary families of models is unknown.

In this paper, we have outlined a graphical model-checking procedure in the spirit of Anscombe (1973). One could extend our work to implement a formal hypothesis-testing framework for rejecting the Kingman coalescent model best-fit according to some inference method. Numerical calculations would require higher moments of the branchlength distribution, which may be computationally intractable for large samples. On the other hand, it would be straightforward to develop a bootstrap-style procedure, using coalescent simulations to estimate the variance in test statistics under the fit model. In any case, we believe that it would be useful for simulation packages such as msprime and population genetics analysis libraries to include standard functions for computing the 2-SFS, fPMI, and hiloPMI. We provide such functions in the GitHub repository associated with this paper.

Our coalescent simulations implement a particular version of the multiple-merger coalescent with recombination. However, the precise correspondence between this model and any particular forward-time process is unclear. In order to compare with our numerical calculations based on Birkner et al. (2013), we use the beta coalescent for the distribution of marginal genealogies, but the explicit forward-time models that have been studied by others generate simultaneous multiple mergers. (See e.g., the Durrett and Schweinsberg 2005 model of selective sweeps, but note that our forward-time simulations suggest that this distinction is not important to our main results.) Moreover, 
the long-range correlations observed in Fig. 5 likely depend on an implicit choice regarding the scaling of recombination, coalescent, and multiple-merger rates (Eldon and Wakeley 2006). These issues are poorly understood, and more theoretical work is required to understand the interactions between multiple mergers and recombination.

An interesteing potential future empircal application of our work would be to use fPMI to assess the evidence for variation in multiplemerger coalescence within genomes and between species. For example, one could compute the PPMI in different regions of a large genome and look for a relationship between the strength of non-Kingman coalescence and genomic properties such as the recombination rate and functional density. Alternatively, one could survey multiple species using a data set such as the diversity data compiled by Corbett-Detig et al. (2015). Either would reveal new information about the suitability of population genetic models, and the forces that determine genetic diversity.

\section{Acknowledgements}

D.P.R. was supported by the Chicago Fellows Program of the University of Chicago. J.N. acknowledges support for this work from NIH grants GM108805 and HG007089. M.M.D. acknowledges support from the Simons Foundation (Grant 376196), grant DEB-1655960 from the NSF, and grant GM104239 from the NIH. This work was completed in part with resources provided by the University of Chicago Research Computing Center and Harvard Faculty of Arts and Sciences Research Computing Center. We thank Arjun Biddanda, Maryn Carlson, Ivana Cvijović, Ben Good, Dick Hudson, Evan Koch, Joe Marcus, Richard Neher, Matthias Steinrücken, John Wakeley, and Aleksandra Walczak for helpful discussions and comments on the manuscript.

\section{Competing interests}

The authors have no competing interests.

\section{References}

Anscombe, F. J. (1973). "Graphs in Statistical Analysis". The American Statistician 27.1, pp. 17-21.

Bhaskar, Anand and Yun S Song (2014). "Descartes' Rule of Signs and the Identifiability of Population Demographic Models from Genomic Variation Data". Ann. Stat. 42.6, pp. 2469 2493. 
Bhaskar, Anand, Y X Rachel Wang, and Yun S Song (2015). "Efficient Inference of Population Size Histories and LocusSpecific Mutation Rates from Large-Sample Genomic Variation Data". Genome Res. 25.2, pp. 268-279.

Birkner, Matthias, Jochen Blath, and Bjarki Eldon (2013). "Statistical Properties of the Site-Frequency Spectrum Associated with Lambda-Coalescents". Genetics 195.3, pp. 1037-1053.

Blath, Jochen, Mathias Christensen Cronjäger, Bjarki Eldon, and Matthias Hammer (2016). "The Site-Frequency Spectrum Associated with $\Xi$-Coalescents". Theor. Popul. Biol. 110, pp. 36-50.

Church, Kenneth Ward and Patrick Hanks (1990). "Word Association Norms, Mutual Information, and Lexicography". Comput. Linguist. 16.1, pp. 22-29.

Comeron, Josep M., Ramesh Ratnappan, and Samuel Bailin (2012). "The Many Landscapes of Recombination in Drosophila Melanogaster". PLOS Genetics 8.10, e1002905.

Coop, Graham and Peter Ralph (2012). "Patterns of Neutral Diversity Under General Models of Selective Sweeps". Genetics 192.1, pp. 205-224.

Corbett-Detig, Russell B., Daniel L. Hartl, and Timothy B. Sackton (2015). "Natural Selection Constrains Neutral Diversity across A Wide Range of Species". PLoS Biology 13.4.

Cover, Thomas M. and Joy A. Thomas (1991). Elements of Information Theory. New York, NY, USA: Wiley-Interscience.

Cvijović, Ivana, Benjamin H. Good, and Michael M. Desai (2018). "The Effect of Strong Purifying Selection on Genetic Diversity". Genetics, genetics.301058.2018.

Desai, Michael M., Aleksandra M. Walczak, and Daniel S. Fisher (2013). "Genetic Diversity and the Structure of Genealogies in Rapidly Adapting Populations". Genetics 193.2, pp. 565585.

Donnelly, Peter and Thomas G. Kurtz (1999). "Particle Representations for Measure-Valued Population Models". The Annals of Probability 27.1, pp. 166-205.

Durrett, Rick and Jason Schweinsberg (2005). "A Coalescent Model for the Effect of Advantageous Mutations on the Genealogy of a Population". Stochastic Processes and their Applications 115.10, pp. 1628-1657. 
Eldon, Bjarki (2016). "Inference Methods for Multiple Merger Coalescents". In: Evolutionary Biology: Convergent Evolution, Evolution of Complex Traits, Concepts and Methods. Ed. by Pierre Pontarotti. Cham: Springer International Publishing, pp. 347-371.

Eldon, Bjarki, Matthias Birkner, Jochen Blath, and Fabian Freund (2015). "Can the Site-Frequency Spectrum Distinguish Exponential Population Growth from Multiple-Merger Coalescents?" Genetics 199.3, pp. 841-856.

Eldon, Bjarki and John Wakeley (2006). "Coalescent Processes When the Distribution of Offspring Number Among Individuals Is Highly Skewed". Genetics 172.4, pp. 2621-2633.

Elyashiv, Eyal, Shmuel Sattath, Tina T. Hu, Alon Strutsovsky, Graham McVicker, Peter Andolfatto, Graham Coop, and Guy Sella (2016). "A Genomic Map of the Effects of Linked Selection in Drosophila". PLoS Genetics 12.8.

Etheridge, Alison (2011). "Mutation and Random Genetic Drift". In: Some Mathematical Models from Population Genetics: École d'Été de Probabilités de Saint-Flour XXXIX-2009. Ed. by Alison Etheridge. Lecture Notes in Mathematics. Berlin, Heidelberg: Springer Berlin Heidelberg, pp. 5-32.

Ferretti, Luca, Alexander Klassmann, Emanuele Raineri, Sebastián E. Ramos-Onsins, Thomas Wiehe, and Guillaume Achaz (2018). "The Neutral Frequency Spectrum of Linked Sites". Theoretical Population Biology.

Fu, Y X (1995). "Statistical Properties of Segregating Sites". Theor. Popul. Biol. 48.2, pp. 172-197.

Garud, Nandita R and Dmitri A Petrov (2016). "Elevated Linkage Disequilibrium and Signatures of Soft Sweeps Are Common in Drosophila Melanogaster". Genetics 203.2, pp. 863880.

Good, Benjamin H., Aleksandra M. Walczak, Richard A. Neher, and Michael M. Desai (2014). "Genetic Diversity in the Interference Selection Limit". PLOS Genetics 10.3, e1004222.

Griffiths, R C and Simon Tavaré (1998). "The Age of a Mutation in a General Coalescent Tree". Communications in Statistics. Stochastic Models 14.1-2, pp. 273-295.

Griffiths, R. C. and S. Tavaré (1994). "Sampling Theory for Neutral Alleles in a Varying Environment". Philosophical Trans- 
actions of the Royal Society of London. Series B, Biological Sciences 344.1310, pp. 403-410.

Gutenkunst, Ryan N., Ryan D. Hernandez, Scott H. Williamson, and Carlos D. Bustamante (2009). "Inferring the Joint Demographic History of Multiple Populations from Multidimensional SNP Frequency Data". PLOS Genetics 5.10, e1000695.

Hahn, M.W. (2018). Molecular Population Genetics. Sinauer Series. Oxford University Press.

Hudson, R. R. (1983). "Properties of a Neutral Allele Model with Intragenic Recombination". Theoretical Population Biology 23.2 , pp. $183-201$.

Hudson, Richard R. (2001). "Two-Locus Sampling Distributions and Their Application". Genetics 159.4, pp. 1805-1817.

- (2002). "Generating Samples under a Wright-Fisher Neutral Model of Genetic Variation". Bioinformatics 18.2, pp. 337338.

Keightley, Peter D., Rob W. Ness, Daniel L. Halligan, and Penelope R. Haddrill (2014). "Estimation of the Spontaneous Mutation Rate per Nucleotide Site in a Drosophila Melanogaster Full-Sib Family". Genetics 196.1, pp. 313-320.

Kelleher, Jerome, Alison M. Etheridge, and Gilean McVean (2016). "Efficient Coalescent Simulation and Genealogical Analysis for Large Sample Sizes". PLOS Computational Biology 12.5, e1004842.

Kern, Andrew D. and Matthew W. Hahn (2018). "The Neutral Theory in Light of Natural Selection". Molecular Biology and Evolution 35.6, pp. 1366-1371.

Kingman, J. F. C. (1982a). "On the Genealogy of Large Populations". Journal of Applied Probability 19.A, pp. 27-43.

- (1982b). "The Coalescent". Stochastic Processes and their Applications 13.3, pp. 235-248.

Lack, Justin B, Charis M Cardeno, Marc W Crepeau, William Taylor, Russell B Corbett-Detig, Kristian A Stevens, Charles H Langley, and John E Pool (2015). "The Drosophila Genome Nexus: A Population Genomic Resource of 623 Drosophila Melanogaster Genomes, Including 197 from a Single Ancestral Range Population". Genetics 199.4, pp. 1229-1241.

Langley, Charles H., Marc Crepeau, Charis Cardeno, Russell Corbett-Detig, and Kristian Stevens (2011). "Circumventing Heterozygosity: Sequencing the Amplified Genome of a Single 
Haploid Drosophila Melanogaster Embryo". Genetics 188.2, pp. 239-246.

Li, Heng and Richard Durbin (2011). "Inference of Human Population History from Individual Whole-Genome Sequences". Nature 475.7357, pp. 493-496.

Messer, Philipp W. (2013). "SLiM: Simulating Evolution with Selection and Linkage". Genetics 194.4, pp. 1037-1039.

Möhle, Martin and Serik Sagitov (2001). "A Classification of Coalescent Processes for Haploid Exchangeable Population Models". The Annals of Probability 29.4, pp. 1547-1562.

Myers, Simon, Charles Fefferman, and Nick Patterson (2008). "Can One Learn History from the Allelic Spectrum?" Theoretical Population Biology 73.3, pp. 342-348.

Neher, R. A. and O. Hallatschek (2013). "Genealogies of Rapidly Adapting Populations". Proceedings of the National Academy of Sciences 110.2, pp. 437-442.

Nicolaisen, Lauren E. and Michael M. Desai (2012). "Distortions in Genealogies Due to Purifying Selection". Molecular Biology and Evolution 29.11, pp. 3589-3600.

Pitman, Jim (1999). "Coalescents With Multiple Collisions". The Annals of Probability 27.4, pp. 1870-1902.

Ragsdale, Aaron P and Ryan N Gutenkunst (2017). "Inferring Demographic History Using Two-Locus Statistics". Genetics 206.2, pp. 1037-1048.

Rödelsperger, Christian, Richard A. Neher, Andreas M. Weller, Gabi Eberhardt, Hanh Witte, Werner E. Mayer, Christoph Dieterich, and Ralf J. Sommer (2014). "Characterization of Genetic Diversity in the Nematode Pristionchus Pacificus from Population-Scale Resequencing Data". Genetics 196.4, pp. 1153-1165.

Rosenberg, Noah A. and Magnus Nordborg (2002). "Genealogical Trees, Coalescent Theory and the Analysis of Genetic Polymorphisms". Nature Reviews Genetics 3.5, pp. 380-390.

Sagitov, Serik (1999). "The General Coalescent with Asynchronous Mergers of Ancestral Lines". Journal of Applied Probability 36.4, pp. 1116-1125.

- (2003). "Convergence to the Coalescent with Simultaneous Multiple Mergers". Journal of Applied Probability 40.4, pp. 839854 . 
Sargsyan, Ori and John Wakeley (2008). "A Coalescent Process with Simultaneous Multiple Mergers for Approximating the Gene Genealogies of Many Marine Organisms". Theor. Popul. Biol. 74.1, pp. 104-114.

Schraiber, Joshua G. and Joshua M. Akey (2015). "Methods and Models for Unravelling Human Evolutionary History". $\mathrm{Na}$ ture Reviews Genetics 16.12, pp. 727-740.

Schrider, Daniel R., Alexander G. Shanku, and Andrew D. Kern (2016). "Effects of Linked Selective Sweeps on Demographic Inference and Model Selection". Genetics 204.3, pp. 12071223.

Schweinsberg, Jason (2000). "Coalescents with Simultaneous Multiple Collisions". Electronic Journal of Probability 5.

- (2003). "Coalescent Processes Obtained from Supercritical Galton-Watson Processes". Stochastic Processes and their Applications 106.1, pp. 107-139.

Seger, Jon, Wendy A. Smith, Jarom J. Perry, Jessalynn Hunn, Zofia A. Kaliszewska, Luciano La Sala, Luciana Pozzi, Victoria J. Rowntree, and Frederick R. Adler (2010). "Gene Genealogies Strongly Distorted by Weakly Interfering Mutations in Constant Environments". Genetics 184.2, pp. 529545 .

Sella, Guy, Dmitri A. Petrov, Molly Przeworski, and Peter Andolfatto (2009). "Pervasive Natural Selection in the Drosophila Genome?" PLOS Genetics 5.6, e1000495.

Sheehan, Sara, Kelley Harris, and Yun S. Song (2013). "Estimating Variable Effective Population Sizes from Multiple Genomes: A Sequentially Markov Conditional Sampling Distribution Approach". Genetics 194.3, pp. 647-662.

Spence, Jeffrey P, John A Kamm, and Yun S Song (2016). "The Site Frequency Spectrum for General Coalescents". Genetics 202.4, pp. 1549-1561.

Steinrücken, Matthias, Matthias Birkner, and Jochen Blath (2013). "Analysis of DNA Sequence Variation within Marine Species Using Beta-Coalescents". Theoretical Population Biology. Coalescent Theory 87, pp. 15-24.

Tajima, F. (1989). "Statistical Method for Testing the Neutral Mutation Hypothesis by DNA Polymorphism." Genetics 123.3, pp. 585-595. 
Tajima, Fumio (1983). "Evolutionary Relationship of DNA Sequences in Finite Populations". Genetics 105.2, pp. 437-460.

Terhorst, Jonathan, John A. Kamm, and Yun S. Song (2017). "Robust and Scalable Inference of Population History from Hundreds of Unphased Whole Genomes". Nature Genetics 49.2, pp. 303-309.

Vitti, Joseph J., Sharon R. Grossman, and Pardis C. Sabeti (2013). "Detecting Natural Selection in Genomic Data". Annual Review of Genetics 47.1, pp. 97-120.

Wakeley, John (2009). Coalescent Theory: An Introduction. Greenwood Village, Colorado: Roberts \& Company. 352 pp.

Watterson, G. A. (1975). "On the Number of Segregating Sites in Genetical Models without Recombination". Theoretical Population Biology 7.2, pp. 256-276.

Xie, Xiaohui (2011). "The Site-Frequency Spectrum of Linked Sites". Bulletin of Mathematical Biology 73.3, pp. 459-494.

Živković, Daniel and Thomas Wiehe (2008). "Second-Order Moments of Segregating Sites Under Variable Population Size". Genetics 180.1, pp. 341-357. 


\section{Supplementary Figures and Tables}
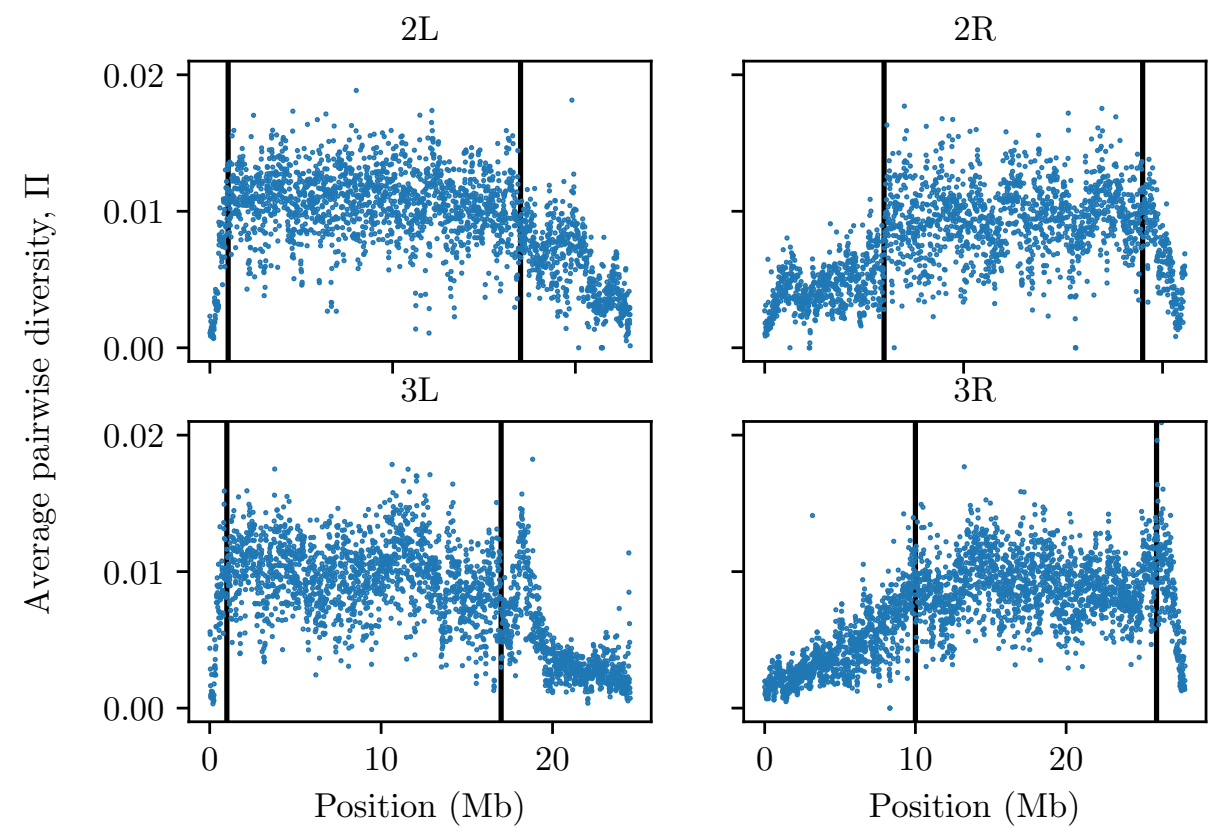

Figure S1: Average pairwise diversity, $\Pi$, of 100 haploid samples from DPGP3 panel. Each point represents the average over a $10 \mathrm{~Kb}$ window. Vertical lines show the boundaries of the central regions defined in Table S1.

Table S1: Boundaries of the homogeneous central regions of chromosomes (shown in Fig. S1).

\begin{tabular}{l|r|r} 
Chr. & Start position $(\mathbf{M b})$ & End position $(\mathbf{M b})$ \\
\hline 2L & 1 & 17 \\
2R & 6 & 19 \\
3L & 1 & 17 \\
3R & 10 & 26
\end{tabular}


Table S2: Fraction of sites with at least 90 genotyped samples

\begin{tabular}{l|r|r} 
Chr. & All sites & Polymorphic sites \\
\hline 2L & 0.913 & 0.924 \\
2R & 0.922 & 0.925 \\
3L & 0.919 & 0.920 \\
3R & 0.933 & 0.936
\end{tabular}

Table S3: Piecewise-constant model parameters fit to the SFS of each chromosome arm (see Eq. (10), $N_{\text {anc }}=3 \times 10^{5}$ ).

\begin{tabular}{l|r|r|r|r} 
Chr. & $N_{1}\left(\times 10^{5}\right)$ & $N_{2}\left(\times 10^{5}\right)$ & $t_{1}\left(\times 10^{4}\right)$ & $t_{2}\left(\times 10^{4}\right)$ \\
\hline 2L & 10.7 & 4.6 & 2.8 & 39.9 \\
2R & 9.1 & 3.9 & 1.6 & 8.8 \\
3L & 7.3 & 3.8 & 1.3 & 6.4 \\
3R & 9.7 & 5.1 & 2.2 & 8.6
\end{tabular}

